\title{
Implementation and Validation of an Impedance Eduction Technique
}

\author{
W. R. Watson, M. G. Jones ${ }^{\dagger}$ and C. H. Gerhold \\ NASA Langley Research Center, Hampton, Virginia 23681-2199, USA
}

\begin{abstract}
Implementation of a pressure gradient method of impedance eduction in two NASA Langley flow ducts is described. The Grazing Flow Impedance Tube only supports plane-wave sources, while the Curved Duct Test Rig supports sources that contain higher-order modes. Multiple exercises are used to validate this new impedance eduction method. First, synthesized data for a hard wall insert and a conventional liner mounted in the Grazing Flow Impedance Tube are used as input to the two impedance eduction methods, the pressure gradient method and a previously validated wall pressure method. Comparisons between the two results are excellent. Next, data measured in the Grazing Flow Impedance Tube are used as input to both methods. Results from the two methods compare quite favorably for sufficiently low Mach numbers but this comparison degrades at Mach 0.5, especially when the hard wall insert is used. Finally, data measured with a hard wall insert mounted in the Curved Duct Test Rig are used as input to the pressure gradient method. Significant deviation from the known solution is observed, which is believed to be largely due to 3-D effects in this flow duct. Potential solutions to this issue are currently being explored.
\end{abstract}

\section{Nomenclature}

$A_{m n}^{ \pm}, B_{m n}^{ \pm} \quad=$ mode coefficients upstream and downstream of liner test section (Pa)

$c_{0}, M_{0}, \rho_{0}=$ speed of sound $(\mathrm{m} / \mathrm{s})$, uniform flow Mach number, and mean static density $\left(\mathrm{kg} / \mathrm{m}^{3}\right)$

$E, f, t \quad=$ transcendental function, source frequency $(\mathrm{Hz})$, and time (s)

$H, W, L \quad=$ height, width, and length of duct (m)

$i \quad=\sqrt{-1}$, unit imaginary number

$k, K_{m n}^{ \pm} \quad=$ freespace and axial wavenumbers $\left(\mathrm{m}^{-1}\right)$

$L_{1}, L_{2} \quad=$ axial locations of the leading and trailing edges of liner (m)

$N H, N V \quad=$ number of horizontal and vertical hard wall duct modes

$n x, n z \quad=$ number of grid points in the $x$ and $z$ directions of grid

$p, p_{m} \quad=3 \mathrm{D}$ and quasi-3D acoustic pressure fields $(\mathrm{Pa})$

$Q_{m}, S_{m} \quad=$ source and termination plane acoustic pressure profiles $(\mathrm{Pa})$

$z, x, y=$ axial, vertical, and horizontal coordinates $(\mathrm{m})$

$X_{m n}, \alpha_{m n}, \lambda_{n}=$ mode shape, complex coefficient, and mode eigenvalue $\left(\mathrm{m}^{-1}\right)$ for vertical mode

$\beta_{0}, \beta_{H} \quad=$ normalized acoustic admittance of lower and upper wall liner

$\zeta_{0}, \zeta_{H} \quad=$ normalized acoustic impedance of lower and upper wall liner

$\eta, \xi=$ unconstrained design variables for optimization algorithm

$\theta_{0}, \theta_{H} \quad=$ normalized acoustic resistance of lower and upper wall liner

$\chi_{0}, \chi_{H} \quad=$ normalized acoustic reactance of lower and upper wall liner

$\kappa_{0}, \sigma_{0} \quad=$ normalized acoustic conductance and susceptance of lower wall liner

$\phi, \omega,\|\| \quad=$ objective function $(\mathrm{Pa} / \mathrm{m})$, circular harmonic frequency $\left(\mathrm{s}^{-1}\right)$, and complex absolute value

${ }^{*}$ Senior Research Scientist, Research Directorate, Computational AeroSciences Branch, Liner Physics Group, Mail Stop 128, Willie.R.Watson@NASA.Gov, (757)-864-5290, AIAA Associate Fellow.

${ }^{\dagger}$ Senior Research Scientist, Research Directorate, Structural Acoustics Branch, Head Liner Physics Group, Mail Stop 463, Michael.G.Jones@NASA.Gov, (757)-864-5272, AIAA Associate Fellow.

${ }^{\ddagger}$ Senior Research Scientist, Research Directorate, Aeroacoustics Branch, Mail Stop 463, Carl.H.Gerhold@NASA.Gov, (757)-864-5279, AIAA Associate Fellow. 


$$
\begin{aligned}
& \text { Superscripts: } \\
& +,-
\end{aligned}=\text { right and left moving wave indicators }
$$

$$
\begin{aligned}
& \text { Subscripts: } \\
& \begin{array}{ll}
m, n & =\text { horizontal and vertical mode orders } \\
I, J & =\text { axial and vertical grid line counters }
\end{array}
\end{aligned}
$$

\section{Introduction}

As part of NASA's Fundamental Aeronautics Program, focused efforts are currently underway to develop concepts to confine subsonic aircraft objectionable noise within the boundaries of the airport. Although acoustic liners mounted in the aircraft engine nacelles provide a significant portion of the current fan-noise reduction, they must be further optimized if this goal is to be achieved. This will require more detailed understanding of an intrinsic parameter of the nacelle liner referred to as the locally-reacting acoustic impedance. This intrinsic parameter is dependent on sound pressure level and grazing flow velocity. Even after four decades of research in impedance measurement technology, accurate and reliable tools for a direct measurement of this intrinsic parameter have proved challenging.

As an alternative to direct measurement of the locally-reacting acoustic impedance, various numerical methods (which are used in conjunction with measurements from experimental facilities) have been developed. The numerical methods $^{1-12}$ include codes based on single mode, multi-mode, and finite element analyses. Of these, finite element codes developed at the NASA Langley Research Center (LaRC) have been thoroughly tested in the open duct acoustics literature $^{2,7,9}$ and have been demonstrated to provide quality results when used in conjunction with measurements acquired in the NASA LaRC Grazing Flow Impedance Tube (GFIT). A schematic of the GFIT's flow duct is given in Fig. 1. The figure shows the liner test section (i.e., the portion of the flow duct that contains the liner) along with the hard wall sections of duct upstream and downstream of the liner test section. The mean flow is directed from left to right along the axis of the duct. The locally-reacting acoustic impedance is educed for test liners in the GFIT using a procedure that has been well established. This procedure ${ }^{2}$ uses the acoustic pressure measured by microphones flushmounted in the hard wall opposite the test liner (see Fig. 1) to educe the test liner impedance and has been referred to in subsequent works as the wall pressure method (WPM) of impedance eduction.

A recent paper ${ }^{13}$ gives a description of the design and development of the Curved Duct Test Rig (CDTR). This rig was initially designed as a test bed to quantify the performance of current liner designs and to develop new concepts for efficient duct liner treatments in the presence of grazing flow and wall curvature. Although impedance eduction at LaRC is currently performed using acoustic measurements acquired in the GFIT, the decision was made in 2010 to acquire the capability to perform impedance eduction using measurements obtained in the CDTR. This decision was made because: (1) flow profiles acquired in the CDTR are typically much closer to those of a full-scale engine than those acquired in the much smaller GFIT, (2) the CDTR waveguide is closer to the size of the aft bypass duct of a business jet, and (3) higher-order modes can be controlled in the CDTR. Figure 2 gives a schematic of the flow duct for the CDTR with straight walls. Shown in the figure are the liner test section (rotated ninety degrees for convenience) and the hard wall sections upstream and downstream of the liner test section. Note that the liner test section contains test liners on opposite walls and is not populated with flush mounted microphones as in the GFIT.

In a previous paper, ${ }^{14}$ it was demonstrated that the GFIT impedance eduction method could be successful if the impedance eduction data were acquired in a hard wall adjacent to the test liner (i.e., the left or right side wall in Fig. 1). While plans are to investigate this method of impedance eduction in the CDTR, the hardware required for obtaining the necessary sidewall measurements is not currently in place. However, in a recent paper, ${ }^{15}$ a new numerical procedure for educing the impedance of test liners located in a duct with grazing flow was presented. This procedure, referred to as the pressure gradient method (PGM) of impedance eduction has the advantage that it is applicable to ducts with test liners on more than one wall as illustrated in Fig. 2, is less cumbersome than the WPM (i.e., no microphones need be installed in the liner test section), and can be applied using the instrumentation already installed in the CDTR. Further, in Ref. 15 the PGM was successfully tested in a duct with small cross-sectional dimensions (i.e., the GFIT) using synthesized data for which only plane waves are cut on in the hard wall sections of the duct. Whether the PGM can be successfully applied in a duct with higher-order modes cut on or using measured data remains an open question.

The current paper has four primary purposes: (1) to describe the implementation and validation of the PGM to a duct with small cross-sectional dimensions (i.e., the GFIT), (2) to describe the implementation of the PGM to a much larger duct (i.e., the CDTR) for which higher-order modes are cut on, (3) to present a consistency check on the implementation of the PGM in the larger duct by demonstrating that it reproduces the same impedance as measured in 
the GFIT when synthesized data is applied, and (4) to present the results of a validation exercise that uses measured data. The remainder of this paper is organized as follows. Section II presents the governing differential equations and boundary conditions, Section III provides a description of the pressure gradient method (PGM) of impedance eduction, Section IV describes how the PGM is implemented in the GFIT and CDTR, results are presented in section V, and the primary conclusions relevant to the study are provided in section VI.

\section{Governing Equations and Boundary Conditions}

Schematics of the flow duct for the GFIT and the CDTR (rotated ninety degrees for convenience) are given in Figs. 1 and 2, respectively. Here, the mean flow is directed from left to right along the axis of the duct and for purposes of this investigation there is no wall curvature in the CDTR (Fig. 2). The same orthogonal coordinate system is used to describe both flow ducts in which $z$ is the axial coordinate, $y$ is the horizontal coordinate, $x$ is the vertical coordinate, and the source and termination planes (of each flow duct) are located at $z=0$ and $z=L$, respectively. In the current setup, the test liner for the GFIT is located on the upper wall, whereas the CDTR may contain test liners on the upper and lower walls. Each liner is assumed to be locally reacting with uniform impedance. The left and right sidewalls are rigid in both duct configurations with the leading and trailing edges of each liner located at $z=L_{1}$ and $z=L_{2}$, respectively.

The analysis used in this paper makes three simplifying assumptions: (1) the flow field in the duct can be decomposed into an acoustic and mean flow field and the mean velocity field has only a uniform axial component, (2) the acoustic field is linear and time-periodic, and (3) the sidewalls of the flow duct are rigid. Under these simplifying assumptions, the acoustic pressure field in the flow duct can be decomposed into a series of hard wall duct modes of the form

$$
p(z, x, y)=\sum_{m=0}^{\infty} \cos \left(\frac{m \pi y}{W}\right) p_{m}(z, x)
$$

where a time dependence of the form $e^{i \omega t}$ has been assumed. The quasi-3D acoustic pressure field, $p_{m}(z, x)$, in Eq. (1) can be shown to satisfy the convected Helmholtz equation

$$
\left(1-M_{0}^{2}\right) \frac{\partial^{2} p_{m}(z, x)}{\partial z^{2}}+\frac{\partial^{2} p_{m}(z, x)}{\partial x^{2}}-2 i k M_{0} \frac{\partial p_{m}(z, x)}{\partial z}+\left[k^{2}-\left(\frac{m \pi}{W}\right)^{2}\right] p_{m}(z, x)=0
$$

Note that Eq. (2) neglects the effects of the mean boundary layer and will become less accurate at the higher flow speeds where gradient in the mean boundary layer are more important. Subsequently, the accuracy of the impedances educed using the method proposed in this paper is expected to degrade at the higher flow speed.

The quasi-3D convected Helmholtz equation (Eq. (2)) is solved subject to the sound source

$$
p_{m}(0, x)=S_{m}(x)
$$

and termination plane boundary condition

$$
p_{m}(L, x)=Q_{m}(x)
$$

Finally, each acoustically treated wall of the duct will require an impedance boundary condition. The boundary condition for a locally-reacting impedance wall has been derived in a previous paper. ${ }^{16}$ When written along the top wall the locally-reacting wall impedance boundary condition is

$$
-\frac{\partial p_{m}(z, H)}{\partial x}=i k\left[\frac{p_{m}(z, H)}{\zeta_{H}}\right]+2 M_{0} \frac{\partial}{\partial z}\left[\frac{p_{m}(z, H)}{\zeta_{H}}\right]+\frac{M_{0}^{2}}{i k} \frac{\partial^{2}}{\partial z^{2}}\left[\frac{p_{m}(z, H)}{\zeta_{H}}\right]
$$

and along the lower wall the boundary condition is

$$
\frac{\partial p_{m}(z, 0)}{\partial x}=i k\left[\frac{p_{m}(z, 0)}{\zeta_{0}}\right]+2 M_{0} \frac{\partial}{\partial z}\left[\frac{p_{m}(z, 0)}{\zeta_{0}}\right]+\frac{M_{0}^{2}}{i k} \frac{\partial^{2}}{\partial z^{2}}\left[\frac{p_{m}(z, 0)}{\zeta_{0}}\right]
$$

In Eqs. (5) and (6), $\zeta_{0}$ and $\zeta_{H}$ are the unknown normalized impedances of the test liners located on the lower and upper wall, respectively. Further, the reciprocal of the normalized impedances are taken as zero along the hard wall portion of the duct wall upstream and downstream of the liner test section. Throughout this paper all impedances are normalized by dividing by the characteristic impedance, $\rho_{0} c_{0}$, of the air in the duct. 
For specified wall impedances $\zeta_{0}$ and $\zeta_{H}$, Eqs. (2)-(6) constitute a well-posed boundary value problem (BVP) that can be solved to determine a unique set of acoustic pressure gradient profiles ( $\frac{\partial p_{m}(0, x)}{\partial z}$ and $\left.\frac{\partial p_{m}(L, x)}{\partial z}\right)$, normal to the source and duct termination planes. The uniqueness of these acoustic pressure gradient profiles provides the basis for the PGM impedance eduction method that is the subject of the following section. Closed-form solutions for these profiles are not yet available for arbitrary source and termination plane acoustic pressure profiles and wall impedances. In this paper, a finite element method that employs cubic Hermite polynomial basis functions ${ }^{2}$ is used to numerically extract these profiles.

\section{The Impedance Eduction Algorithm}

The PGM has been described in an earlier paper. ${ }^{15}$ Therefore only enough is presented in this section to provide continuity with the previous paper and to further motivate the methods and goals of this investigation. Because the PGM uses an optimization algorithm as a workhorse to extract the impedance, the optimization algorithm is briefly described.

\section{A. Brief Description of the PGM}

The PGM may be summarized as follows:

1. Measure the mean flow field, the source and termination plane acoustic pressure profiles, and the acoustic pressure gradient profiles normal to the source and duct termination planes.

2. Using initial guesses for the test liner impedances, $\zeta_{0}$ and $\zeta_{H}$, solve the BVP defined by Eqs. (2)-(6) to obtain a unique set of acoustic pressure gradient profiles normal to the source and duct termination planes. Because exact solutions to the BVP are generally not available, a numerical method is required to obtain these profiles.

3. Compare the measured acoustic pressure gradient profiles at the source and duct termination planes to those obtained from the numerical solution to the BVP. If the numerically computed and measured acoustic pressure gradient profiles match (to within some tolerance) then the initial guesses for the test liner impedances are the impedances of the test liners.

4. If the numerically computed and measured acoustic pressure gradient profiles do not match, then the unknown impedances, $\zeta_{0}$ and $\zeta_{H}$, are iteratively updated using an automated gradient-based optimizer until the measured and numerically computed acoustic pressure gradient profiles match (to within some tolerance). The resulting impedances are the unknown impedances of the test liners.

It is noted that the automated gradient-based optimizer iterates on the impedances by minimizing the objective function, $\phi\left(\zeta_{0}, \zeta_{H}\right)$, that is defined as

$$
\phi\left(\zeta_{0}, \zeta_{H}\right)=\sqrt{\sum_{J=1}^{n x}\left\|\left[\frac{\partial \bar{p}_{m}\left(0, x_{J}\right)}{\partial z}-\frac{\partial p_{m}\left(0, x_{J}\right)}{\partial z}\right]\right\|+\left\|\left[\frac{\partial \bar{p}_{m}\left(L, x_{J}\right)}{\partial z}-\frac{\partial p_{m}\left(L, x_{J}\right)}{\partial z}\right]\right\|}
$$

In Eq. (7), $\frac{\partial p_{m}}{\partial z}$, is the normal acoustic pressure gradient obtained from the numerical solution to the BVP and $\frac{\partial \bar{p}_{m}}{\partial z}$ is the normal acoustic pressure gradient obtained from the measurement. The automated gradient-based optimizer that finds the impedances that minimizes the above objective function is one of the most important features of the PGM. Therefore, the strengths and weaknesses of the automated optimizer that is used with the PGM are briefly discussed in the following subsection.

\section{B. The Optimization Algorithm}

The optimization algorithm that is used in this paper is a modification of the Davidon-Fletcher-Powell (DFP) optimization algorithm. The DFP optimization algorithm is a technique for finding the minimum of an objective function such as that defined in Eq. (7). It was first proposed by Davidon ${ }^{17}$ and was later reformulated and popularized by Fletcher and Powell. ${ }^{18}$ The version of the algorithm that is used in this paper has been modified to accept numerical differentiation of the gradient as suggested by Stewart. ${ }^{19}$ This modified algorithm has become quite popular because of its speed and robustness and is referred to here as "Stewart's adaptation of the Davidon-Fletcher-Powell" (SDFP) optimization algorithm. 
The most important assumption made by the SDFP optimization algorithm (that has bearing on results presented in this paper) is the assumption that the objective function can be locally approximated as a quadratic in the region around the minimum point. This optimization method, therefore, has the disadvantage that it may converge to a local minimum and may become stuck in the portion of the impedance plane where the objective function is extremely flat. However, this shortcoming of the SDFP algorithm is more than mitigated by the fact that it converges faster and yields more accurate results than many of its competitors.

A second important issue that affects the accuracy of the SDFP optimization algorithm is that it accepts only real design variables and is designed for unconstrained optimization problems. The normalized impedance of each liner is therefore divided into its normalized resistance $\left(\theta_{0}, \theta_{H}\right)$ and normalized reactance components $\left(\chi_{0}, \chi_{H}\right)$

$$
\begin{gathered}
\zeta_{0}=\theta_{0}+i \chi_{0} \\
\zeta_{H}=\theta_{H}+i \chi_{H}
\end{gathered}
$$

Because the optimization algorithm is unconstrained, the SDFP optimization algorithm allows the design variables to take on values corresponding to any real number, $-\infty \leq\left(\theta_{0}, \theta_{H}\right) \leq \infty,-\infty \leq\left(\chi_{0}, \chi_{H}\right) \leq \infty$. From a practical consideration of the geometry of nacelle liners used in aircraft engine noise applications (i.e., hole size, face sheet thickness, open area ratio, depth of the honeycomb core, etc.), the normalized resistance and reactance of the liner fall within the range, $0 \leq\left(\theta_{0}, \theta_{H}\right) \leq 10,-10 \leq\left(\chi_{0}, \chi_{H}\right) \leq 10$. The capability to constrain both the normalized resistance and reactance to physically realizable values is implemented in this study. This is done in the following manner. Let the design variables used by the SDFP optimization algorithm be denoted by $\xi$ and $\eta$. Now introduce the following constraints into SDFP:

$$
\begin{gathered}
\theta=5[1+\cos (\xi)] \\
\chi=10 \cos (\eta)
\end{gathered}
$$

Note that as the design variables $\xi$ and $\eta$ take on any real number, the normalized resistance and reactance of the liner will take on only physically realizable values (i.e., $0 \leq \theta \leq 10,-10 \leq \chi \leq 10$ ). Finally, a stopping criterion (i.e., tolerance) of $1 \times 10^{-8}$ is used to terminate the SDFP optimization algorithm.

\section{Implementation of the Impedance Eduction Method}

The PGM has been implemented in both the GFIT and the CDTR. Because only plane waves are cut on in the hard wall sections of the GFIT while higher order modes are cut on in the CDTR, the implementation of the PGM method is different in both rigs. Four acoustic measurements are needed to implement the PGM. The first two measurements are the source and termination plane acoustic pressure profiles $\left(S_{m}(x)\right.$ and $\left.Q_{m}(x)\right)$. The next two acoustic measurements are the source and termination plane acoustic pressure gradient profiles $\left(\frac{\partial \bar{p}_{m}(0, x)}{\partial z}\right.$ and $\left.\frac{\partial \bar{p}_{m}(L, x)}{\partial z}\right)$. The following two subsections describe how the PGM is implemented in both rigs to acquire these two sets of data.

\section{A. Implementation in the GFIT}

In the GFIT, two microphones (not shown in Fig. 1) are flush-mounted in the hard wall section upstream of the liner test section to provide a measurement of the acoustic pressure gradient profile at the source plane. These two microphones are flush-mounted in the lower wall (i.e., at $x=x_{1}=0$ ) at points in the finite element grid. The first microphone is located at the source plane (i.e., $z=z_{1}=0$ ) and the second microphone is located one grid point to the right of the source plane (i.e., $z=z_{2}$ ). Therefore the measured acoustic pressure gradient profile normal to the source plane is approximated using the forward difference expression

$$
\frac{\partial \bar{p}_{m}(0, x)}{\partial z}=\frac{\left[p_{m}\left(z_{2}, x\right)-p_{m}\left(z_{1}, x\right)\right]}{\left(z_{2}-z_{1}\right)}
$$

Because only plane waves are cut on in the GFIT, the acoustic pressure profiles $\left(p_{m}\left(z_{2}, x\right)\right.$ and $\left.p_{m}\left(z_{1}, x\right)\right)$ are independent of the vertical coordinate, $x$. Therefore, $p_{m}\left(z_{2}, x\right)$ and $p_{m}\left(z_{1}, x\right)$ are obtained from the acoustic pressure measurements obtained from the two lower wall microphones (i.e., $p_{m}\left(z_{2}, x\right)=p_{m}\left(z_{2}, x_{1}\right)$ and $p_{m}\left(z_{1}, x\right)=p_{m}\left(z_{1}, x_{1}\right)$ ) and only the zeroth order horizontal mode (i.e., $m=0$ ) need be analyzed.

A method similar to that discussed in the preceding paragraph is used to approximate the acoustic pressure gradient profile normal to the duct termination plane. Thus, the backward difference expression

$$
\frac{\partial \bar{p}_{m}(L, x)}{\partial z}=\frac{\left[p_{m}\left(z_{n z}, x\right)-p_{m}\left(z_{n z-1}, x\right)\right]}{\left(z_{n z}-z_{n z-1}\right)}
$$


is used to approximate the termination plane acoustic pressure gradient. In Eq. (13), $p_{m}\left(z_{n z}, x\right)$ and $p_{m}\left(z_{n z-1}, x\right)$ are the complex acoustic pressures measured by two lower wall microphones located at $z=z_{n z-1}$ and $z=z_{n z}=L$, respectively. Here $n z$ is the number of axial grid lines in the finite element grid. Just as at the source plane, this acoustic pressure gradient profile is also constant across the duct and only the zeroth order horizontal mode (i.e., $m=0$ ) need be analyzed. Finally, the measured lower wall acoustic pressure is used as the source- and termination-plane acoustic pressure profiles

$$
\begin{aligned}
& p_{m}(0, x)=p_{m}\left(z_{1}, x_{1}\right) \\
& p_{m}(L, x)=p_{m}\left(z_{n z}, x_{1}\right)
\end{aligned}
$$

where $p_{m}\left(z_{1}, x_{1}\right)$ and $p_{m}\left(z_{n z}, x_{1}\right)$ are obtained from the flush-mounted lower wall microphones located at the source (i.e., $z=0)$ and termination $(z=L)$ planes, respectively. Further, because only plane waves are cut on in the GFIT, only the zeroth order horizontal mode (i.e., $m=0$ ) need be analyzed.

\section{B. Implementation in the CDTR}

Because the CDTR contains higher-order modes that are cut on in the hard wall sections upstream and downstream of the test section, the implementation of the PGM to the CDTR is considerably more complicated than the GFIT. In the CDTR there are two measurement sections with microphones, one upstream of the liner test section and one downstream. ${ }^{13}$ The microphone arrays are identical in both sections, each containing 47 flush-mounted microphones. The microphones in each array are strategically located around the circumference of the duct at several axial locations. The locations are selected to maximize resolution of the modal content of the acoustic field. ${ }^{13}$ Data acquired at these two microphone arrays are used to obtain the necessary data for the PGM.

Let $p^{u}\left(z_{I}, x_{I}, y_{I}\right)$ and $p^{d}\left(z_{I}, x_{I}, y_{I}\right)$ represent the measured acoustic pressures at microphone $I$ in the upstream $(0 \leq$ $\left.z_{I} \leq L_{1}\right)$ and downstream $\left(L_{2} \leq z_{I} \leq L\right)$ microphone array, respectively. The acoustic pressures at microphone, $I$, are expanded into a series of hard wall duct modes in the hard wall sections upstream and downstream of the liner test section as follows

$$
\begin{gathered}
p^{u}\left(z_{I}, x_{I}, y_{I}\right)=\sum_{m=0}^{N H-1} \sum_{n=0}^{N V-1}\left[A_{m n}^{+} e^{i K_{m n}^{+} z_{I}}+A_{m n}^{-} e^{i K_{m n}^{-} z_{I}}\right] \cos \left(\frac{n \pi x_{I}}{H}\right) \cos \left(\frac{m \pi y_{I}}{W}\right), \quad\left(0 \leq z_{I} \leq L_{1}\right) \\
p^{d}\left(z_{I}, x_{I}, y_{I}\right)=\sum_{m=0}^{N H-1} \sum_{n=0}^{N V-1}\left[B_{m n}^{+} e^{i K_{m n}^{+} z_{I}}+B_{m n}^{-} e^{i K_{m n}^{-} z_{I}}\right] \cos \left(\frac{n \pi x_{I}}{H}\right) \cos \left(\frac{m \pi y_{I}}{W}\right), \quad\left(L_{2} \leq z_{I} \leq L\right) \\
\frac{K_{m n}^{ \pm}}{k}=\frac{M_{0} \mp \sqrt{1-\left(1-M_{0}^{2}\right)\left[(n \pi / k H)^{2}+(m \pi / k W)^{2}\right]}}{\left(1-M_{0}^{2}\right)}
\end{gathered}
$$

Here, $N H$ and $N V$ are the number of horizontal and vertical modes, respectively, in the mode expansion. Generally speaking, $N H$ and $N V$ are chosen so that all cut on modes and a couple of cut off modes are included in the series expansion.

Equations (16) and (17) are a set of linear algebraic equations for each microphone location, $\left(z_{I}, x_{I}, y_{I}\right)$. If the series on the right-hand side of Eqs. (16) and (17) are truncated to the same number of terms as the number of microphones, then the two systems of equations can be solved to obtain the mode coefficients of the right and left traveling acoustic waves (i.e., $A_{m n}^{ \pm}, B_{m n}^{ \pm}$). These known mode coefficients are then used to expand the acoustic pressure field in terms of hard wall duct modes:

$$
\begin{aligned}
& p^{u}(z, x, y)=\sum_{m=0}^{N H-1} \sum_{n=0}^{N V-1}\left[A_{m n}^{+} e^{i K_{m n}^{+} z}+A_{m n}^{-} e^{i K_{m n}^{-} z}\right] \cos \left(\frac{n \pi x}{H}\right) \cos \left(\frac{m \pi y}{W}\right), \quad\left(0 \leq z \leq L_{1}\right) \\
& p^{d}(z, x, y)=\sum_{m=0}^{N H-1} \sum_{n=0}^{N V-1}\left[B_{m n}^{+} e^{i K_{m n}^{+} z}+B_{m n}^{-} e^{i K_{m n}^{-} z}\right] \cos \left(\frac{n \pi x}{H}\right) \cos \left(\frac{m \pi y}{W}\right), \quad\left(L_{2} \leq z \leq L\right)
\end{aligned}
$$

The source and termination plane acoustic pressure profiles $\left(S_{m}(x)\right.$ and $\left.Q_{m}(x)\right)$ needed for the PGM are now determined from the mode series

$$
S_{m}(x)=\sum_{n=0}^{N V-1}\left[A_{m n}^{+}+A_{m n}^{-}\right] \cos \left(\frac{n \pi x}{H}\right)
$$




$$
Q_{m}(x)=\sum_{n=0}^{N V-1}\left[B_{m n}^{+} e^{i K_{m n}^{+} L}+B_{m n}^{-} e^{i K_{m n}^{-} L}\right] \cos \left(\frac{n \pi x}{H}\right)
$$

The measured source and termination plane pressure gradient profiles needed by the PGM are also obtained from these mode series

$$
\begin{gathered}
\frac{\partial \bar{p}_{m}(0, x)}{\partial z}=\sum_{n=0}^{N V-1}\left[i K_{m n}^{+} A_{m n}^{+}+i K_{m n}^{-} A_{m n}^{-}\right] \cos \left(\frac{n \pi x}{H}\right) \\
\frac{\partial \bar{p}_{m}(L, x)}{\partial z}=\sum_{n=0}^{N V-1}\left[i K_{m n}^{+} B_{m n}^{+} e^{i K_{m n}^{+} L}+i K_{m n}^{-} B_{m n}^{-} e^{i K_{m n}^{-} L}\right] \cos \left(\frac{n \pi x}{H}\right)
\end{gathered}
$$

\section{Results and Discussion}

The primary purpose of the results presented in this section is to validate the PGM for a range of test conditions. This is achieved by comparing the educed impedance spectra from the PGM with either known impedance spectra or with impedance spectra educed from the WPM. Impedance eduction data were acquired from three primary sources, (1) a mode solution that is possible when the liner extends from the source to the duct termination planes, (2) data simulated using the finite element solution (i.e., the hard wall sections are included), and (3) data measured in the GFIT and the CDTR (i.e., the hard wall sections are included). Three validation exercises are performed. The first validation exercise is performed with the CDTR geometry, for which higher-order modes are cut on in the hard wall sections over the majority of the frequency range of interest. Results are presented for both a hard wall duct and a conventional liner. For the hard wall duct, the known solution is used to simulate the data for impedance eduction. For the conventional liner, the normalized acoustic impedance of the liner is selected to correspond to a liner previously tested in the GFIT. The eduction data are then simulated from either the mode or finite element solution. Essentially, the first validation exercise constitutes a consistency check to demonstrate that the PGM is capable of properly handling the effects of higher-order duct modes and flow. Following this consistency check, a second validation exercise is performed. The second validation exercise uses measured data from the GFIT (which is capable of acquiring data for both the PGM and the WPM) for plane wave test conditions. The third validation exercise uses measured data from the CDTR for which higher-order modes are cut on.

\section{A. The Consistency Check}

The results of the consistency check are restricted to the geometry of the CDTR for which $L_{1}=0.203 \mathrm{~m}, L_{2}=1.016 \mathrm{~m}$, $L=1.219 \mathrm{~m}, W=0.381 \mathrm{~m}$, and $H=0.152 \mathrm{~m}$. As many as three transverse modes may be cut on for the lowest order horizontal mode in the frequency range of interest (i.e., $0.300 \leq f \leq 2.80 \mathrm{kHz}$ ). Results are obtained for a flow off $\left(M_{0}=0.000\right)$ and two flow on conditions $\left(M_{0}=0.275\right.$, and $\left.M_{0}=0.500\right)$. All results for the consistency check are computed at standard atmospheric conditions $\left(c_{0}=344.28 \mathrm{~m} / \mathrm{s}\right.$ and $\left.\rho_{0}=1.200 \mathrm{~kg} / \mathrm{m}^{3}\right)$.

\section{Hard-Wall Duct Admittance Eductions}

Several examples are now presented to demonstrate the accuracy and robustness of the PGM in hard wall ducts that contain planar and higher-order mode sources. Potential problem areas that may require special treatment are also highlighted. Because the impedance of a hard wall is effectively infinite, the results are presented in terms of the

educed normalized admittance, $\beta_{0}=\frac{1}{\zeta_{0}}=\kappa_{0}+i \sigma_{0}$. The normalized admittance of the upper wall is set to a zero value $\left(\beta_{H}=\frac{1}{\zeta_{H}}=0.0+0.0 i\right)$, and the normalized conductance, $\kappa_{0}$, and susceptance, $\sigma_{0}$, of the lower wall are educed using the PGM. The eduction data for these hard wall consistency checks are simulated from the exact mode solution for outgoing waves in an infinite hard wall duct:

$$
p(z, x, y)=\sum_{m=0}^{N H-1} \sum_{n=0}^{N V-1} A_{m, n}^{+} e^{i K_{m n}^{+} z} \cos \left(\frac{n \pi x}{H}\right) \cos \left(\frac{m \pi y}{W}\right)
$$

Equation (25) is used to extract the source- and termination-plane acoustic pressure and gradient profiles that are required for the PGM. Both cut on and cut off modes are allowed to propagate through the flow duct and the normalized conductance and susceptance values are educed for the following four sources

1. a plane-wave source (i.e., $A_{00}^{+}=20 \mathrm{~Pa}$ ) 
2. the (01) mode (i.e., $A_{01}^{+}=20 \mathrm{~Pa}$ )

3. the (10) mode (i.e., $A_{10}^{+}=20 \mathrm{~Pa}$ )

4. the (11) mode (i.e., $A_{11}^{+}=20 \mathrm{~Pa}$ ).

Prior to performing the impedance eductions, a grid refinement study was conducted using each of the four sound sources to determine the coarsest finite element grid for accurate resolution of the acoustic waves in the hard wall duct. The finite element grid used to perform the impedance eductions was four times denser than the coarsest grid needed for accurate resolution of the acoustic wave in the hard wall duct.

Figure 3a shows the educed normalized conductance and susceptance for the plane wave source (i.e., the (00) hard wall mode) for the flow off $\left(M_{0}=0.000\right)$ and the two flow on conditions $\left(M_{0}=0.275\right.$, and $\left.M_{0}=0.500\right)$. The conductance and susceptance were educed every $0.100 \mathrm{kHz}$ for frequencies ranging from 0.300 to $2.800 \mathrm{kHz}$. As can be seen from the figure, the normalized conductance and susceptance values that were educed with the PGM are in excellent agreement with their expected value of zero. There are 144 test conditions (i.e., three Mach numbers and 48 frequencies) and approximately $10 \%$ of these test conditions had to be restarted because the SDFP did not give a normal return using the initial starting value of $\kappa_{0}=\sigma_{0}=0.500$. In each of these cases the initial starting value had to be reduced below 0.500 . Figure $3 \mathrm{~b}$ shows the educed normalized conductance and susceptance for a higher order mode (i.e. the (01) hard wall mode). The cut on frequency for this mode is approximately $1.200 \mathrm{kHz}$. At frequencies below the cut on frequency, the sound wave is rapidly attenuated, whereas at frequencies above the cut on frequency the sound wave propagates without attenuation. Several observations may be made from Fig. 3b. First, the conductance and susceptance at all frequencies below the cut on frequency are accurately educed. Second, the conductance at frequencies above the cut on frequency is also accurately educed. Third, there are some errors in the educed susceptance at a few of the frequencies just above the cut on frequency. This trend has also been observed in studies with the $\mathrm{WPM}^{7}$ and is due to the fact that above the cut on frequency the sound wave is not attenuated. This leads to an objective function that is extremely flat at these frequencies, causing the educed susceptance to be highly dependent on the starting values. Results for the (01) and (11) hard wall mode are not presented for the sake of brevity. However, the educed conductance and susceptance values for each of these higher-order modes produced trends similar to those observed in Fig. $3 b$.

\section{Soft-Wall Duct Impedance Eductions without Hard Wall Sections}

The results in this subsection test the capability of the PGM to accurately educe the soft-wall impedance. The PGM impedance eduction data were obtained from a mode solution that is possible when the upper and lower wall liners are uniform and cover the full extent of the duct (i.e., extend from $z=0$, to $z=L$ ). In this situation the quasi-3D mode, $p_{m}(z, x)$, has the following closed form solution

$$
p_{m}(z, x)=X_{m n}(x) e^{i K_{m n}^{ \pm} z}
$$

where $n$ is used to order the modes according to their attenuation rates. The vertical modes, $X_{m n}(x)$, are obtained using separation of variables

$$
X_{m n}(x)=\left\{\begin{array}{ll}
\cos \left(\lambda_{n} x\right)+\frac{i k \alpha_{m n}}{\lambda_{n} \zeta_{0}} \sin \left(\lambda_{n} x\right), & \text { for } \lambda_{n} \neq 0 \\
1, & \text { for } \lambda_{n}=0, \text { or } \alpha_{m n}=0
\end{array}\right\}
$$

where $\lambda_{n}$ are complex zeroes of the transcendental function

$$
\begin{gathered}
E\left(k, H, M_{0}, \zeta_{0}, \zeta_{H}, \lambda_{n}\right)=i k H \alpha_{m n} \lambda_{n} H\left(\frac{1}{\zeta_{0}}+\frac{1}{\zeta_{H}}\right)-\left(\frac{k^{2} H^{2}}{\zeta_{0} \zeta_{H}} \alpha_{m n}^{2}+\lambda_{n}^{2} H^{2}\right) \tan \left(\lambda_{n} H\right) \\
\alpha_{m n}=1-2\left(\frac{M_{0} K_{m n}}{k}\right)+\left(\frac{M_{0} K_{m n}}{k}\right)^{2} \\
\frac{K_{m n}^{ \pm}}{k}=\frac{M_{0} \mp \sqrt{1-\left(1-M_{0}^{2}\right)\left[(m \pi / k W)^{2}+\left(\lambda_{n} / k\right)^{2}\right]}}{\left(1-M_{0}^{2}\right)}
\end{gathered}
$$


Note that for a given value of $\lambda_{n}$ the axial propagation constant, $K_{m n}$, that are the complex zeroes of the trancendental function, $E\left(k, H, M_{0}, \zeta_{0}, \zeta_{H}, \lambda_{n}\right)$, has two roots. It is easily shown that right moving waves (i.e., $\left.K_{m n}^{+}\right)$are identified as those modes for which the axial propagation constant has a zero or negative imaginary part and positive real part, respectively. The second root (i.e., $K_{m n}^{-}$) corresponds to left moving waves. In a hard wall duct where, $\lambda_{n}=\frac{n \pi}{H}$, Eq. (30) reduces to the expression given in Eq. (18).

In this paper we are interested in the aft-duct configuration (the sound and the flow are propagating in the same direction) so only $K_{m n}^{+}$is of interest. The method of analysis used here calculates the mode eigenvalues, $\lambda_{n}$, that are the complex zeroes of the transcendental function, $E\left(k, H, M_{0}, \zeta_{0}, \zeta_{H}, \lambda_{n}\right)$, and the corresponding axial propagation constants, $K_{m n}^{+}$. The method used to obtain these roots is an analytic continuation method similar to that used by Snider ${ }^{20}$ where the zero-flow modes are calculated using a matrix method and the flow modes are calculated from the zero-flow modes by gradually increasing the mean flow Mach number from its zero value.

Given the quasi-3D vertical mode, $X_{m n}(x)$, and axial propagation constant, $K_{m n}^{+}$, the closed form solution in Eq. (26) is used to extract the PGM impedance eduction data in the aft-duct configuration for a specified horizontal mode (i.e., $m$ ) and a given vertical mode, $n$

$$
S_{m}(x)=X_{m n}(x), Q_{m}(x)=X_{m n}(x) e^{i K_{m n}^{+} L}, \frac{\partial \bar{p}(0, x)}{\partial z}=i K_{m n}^{+} X_{m n}(x), \frac{\partial \bar{p}(L, x)}{\partial z}=i K_{m n}^{+} X_{m n}(x) e^{i K_{m n}^{+} L}
$$

The PGM impedance eductions are performed for the first two vertical modes $(n=1$ and $n=2)$ where the modes are arranged according to their attenuation rate. The test liner is a single-layer, conventional perforate-over-honeycomb liner. This liner was chosen because it is representative of liners commonly used for the reduction of fan noise in current aircraft engine nacelles. The facesheet for this conventional liner has 0.991-mm-diameter holes, an open area ratio of 0.087 , and a thickness of $0.635 \mathrm{~mm}$. The depth of the cavity is $38.18 \mathrm{~mm}$; this depth was chosen so that the resonant frequency of the liner is in the middle of the frequency range of interest. The impedance of this liner was determined from earlier tests in the GFIT and these impedance values were used in the transcendental equation to extract the vertical modes and impedance eduction data for the PGM.

Figures $4 \mathrm{a}$ and $4 \mathrm{~b}$ compare the normalized resistance and reactance values for the (01), (11), (02), and (12) modes educed by the PGM with that measured in the GFIT for the flow off condition. Results are presented in $0.200 \mathrm{kHz}$ increments. For each sound source, the PGM educed resistance and reactance values are not distinguishable from those obtained using the WPM in the GFIT. Note that when the flow is off, the conventional liner is a low resistance liner whose resistance is nearly independent of frequency (as expected), and the normalized reactance follows a $-\cot (k d)$ (where $d$ is the depth of the cavity) behavior with the resonant frequency (i.e., the frequency at which the reactance approaches zero) at $1.500 \mathrm{kHz}$ (as expected).

The normalized resistance and reactance spectra educed with the WPM and the PGM for a flow on condition (Mach 0.275) and several higher order mode sources (the (01), (11), (02), and (12) modes) are depicted in Figs. 5a and 5b. Educed impedances from both methods (i.e., the WPM and PGM) are in good agreement with each other. The PGM educes the same impedance for each higher order mode source as expected. Both eduction methods show an increase in the normalized resistance as the flow Mach number increases from zero (as expected), but the reactance at the low end of each spectra varies from the $-\cot (k d)$ behavior that was observed for the flow off condition. The differences at the low frequency end of the spectrum were expected and result from the fact that the frequency is approaching an anti-resonant value. This result corresponds to previously reported findings in which the reduced attenuation due to an impedance near anti-resonance causes the "eye" of the objective function contour to be quite diffused (i.e., a large range of impedances produces similar attenuations).

\section{Soft-Wall Duct Impedance Eductions with Hard Wall Sections Upstream and Downstream}

Results presented thus far test the capability of the PGM to accurately educe the conventional liner impedance when a closed-form solution is available to extract the impedance eduction data. However, in real facilities the flow duct contains hard wall sections upstream and downstream of the liner test section so that a closed-form solution is not available. Therefore to test the PGM in the presence of more realistic test conditions, simulations are performed in the CDTR geometry with the hard wall sections included. The axial pressure gradient that is needed to educe the impedance is obtained as follows:

1. The source- and termination-plane acoustic pressure profiles for the CDTR are set to the same profiles that were used in the hard wall example problem.

2. The finite element method is then used (with the conventional liner impedance that was educed in the GFIT) to simulate the axial acoustic pressure gradients needed for the PGM. 
PGM impedance eductions with hard wall sections upstream and downstream of the conventional liner were computed with the flow off (Mach 0.000) and flow on (Mach 0.275). For each sound source (i.e., the (00), (01), (10), and (11)), the normalized resistance and reactance values educed with the PGM matched those educed in the GFIT to two decimal digits of precision except at $0.4 \mathrm{kHz}$ where the attenuation is low. Graphical results for these test cases are not presented in this paper for the sake of brevity.

\section{B. Validation Using Measured Data from the GFIT}

PGM results are now compared to WPM results for cases in which measured data from the GFIT is used. Results are presented for both a hard wall insert and the conventional liner. Data were acquired in the GFIT with the flow off (Mach 0.000) and for two flow on conditions (Mach 0.300 and 0.500). The Mach number for the flow-on condition is the centerline value. The GFIT geometry is rectangular with $H=0.060 \mathrm{~m}, W=0.050 \mathrm{~m}, L_{1}=0.200 \mathrm{~m}, L_{2}=0.610 \mathrm{~m}$, and $L=1.220 \mathrm{~m}$ (see Fig. 1). For the WPM, measured acoustic pressure data were obtained at 53 unevenly spaced points along the bottom wall. For the PGM, a measurement of the acoustic pressure is taken only at the source and exit planes and at $0.030 \mathrm{~m}$ away from these planes (i.e., only four acoustic pressure measurements are required). Because only plane waves are cut on at the frequencies of interest, all acoustic pressure measurements are obtained at the lower hard wall, and these are assumed to be constant in the vertical direction of the duct (i.e., from the lower wall to the upper wall). The hard wall insert is a 12.700 -mm-thick stainless-steel plate and is chosen because it provides a liner for which the normalized admittance, $\beta_{H}$, is known (i.e., $\beta_{H}=0.000+0.000 i$ for a hard wall insert).

\section{Hard Wall Duct Admittance Eductions}

Figure 6 compares the educed normalized conductance and susceptance spectra for the hard wall insert for the flow-off condition. Both the WPM and the PGM are in excellent agreement with each other and with the known conductance and susceptance for a hard wall insert. Figure 7 shows the comparisons for the two flow-on conditions. The agreement between the normalized conductance spectra educed with the WPM and the PGM is quite good for $M_{0}=0.300$ but degrades at $M_{0}=0.500$. The normalized conductance is observed to be fairly close to the expected value of zero for all conditions, but the normalized susceptance deviates from the expected value of zero for both the WPM and PGM for the flow on conditions. This scatter in the susceptance spectra about the expected value of zero increases with the mean flow Mach number. This result is not surprising when one considers that the refractive effects of the mean boundary layer (which are neglected in both the WPM and the PGM) are more important at higher mean flow Mach numbers.

\section{Conventional Liner Impedance Eductions}

Figure 8 compares the educed normalized resistance and reactance spectra for the conventional liner for the flow-off condition (i.e., $M_{0}=0.000$ ). Both the resistance and reactance spectra from the WPM and the PGM method are in excellent agreement with each other. The normalized resistance and reactance spectra educed with the WPM and the PGM for both of the flow-on conditions are depicted in Fig. 9. Educed impedances from both methods (i.e., the WPM and PGM) are in good agreement with each other, especially at the lower Mach numbers.

\section{Results Using CDTR Measurements}

The PGM results for the CDTR were also computed. These were performed as follows:

1. Incident sound waves of $130 \mathrm{~dB}$ (with horizontal mode order, $m_{0}$ ) were generated upstream of the liner test section

2. The sound field at each microphone array in the CDTR was simulated using 20 modes propagating upstream and 20 modes propagating downstream (i.e., $N V=5$ and $N H=4$ in each hard wall section). With this number of modes the microphone acoustic pressure residual (i.e., the difference between the measured and simulated microphone acoustic pressure) was no larger than $10^{-10}$

3. Equations (21)-(24) are used to acquire impedance eduction data using $m=m_{0}$. The quasi-3D equation (Eq. (2)) with $m=m_{0}$ is used in conjunction with the SDFP algorithm to educe the impedance of the liner

Figure 10 shows the educed normalized admittance for the plane wave (i.e., the (00) mode) and the (01) mode for the flow off condition. With few exceptions, the educed conductance is observed to be close to the expected value 
of zero for each of these source conditions. The conductance values educed with the (00) mode deviate from zero at $1.5 \mathrm{kHz}$, while those educed with the (01) mode show scatter at 1.1 and $1.5 \mathrm{kHz}$. On the other hand, the educed susceptance exhibits significantly more scatter than observed for the conductance, for both the (00) and (01) modes. Thus, although the predicted attenuation remains low, the current implementation of the PGM is clearly insufficient for this configuration. All indications are that this problem is caused by the mode expansion at the microphone locations. One solution may be to use the measured acoustic pressure data directly, avoiding the use of the modal expansion. However, this approach has the disadvantage that the solution to the BVP would have to be obtained with a 3-D code because higher order modes are cut on in the CDTR. Potential solutions to this issue, along with other improvements to the PGM, are currently being explored.

\section{Concluding Remarks}

The pressure gradient method (PGM) of impedance eduction has advantages over the wall pressure method (WPM). The primary one is that it does not require the installation of microphones in the liner test section to educe the impedance. This paper has tested the PGM over a range of test conditions using eduction data that were (1) obtained from a closed-form solution, (2) synthesized from a finite element method, and (3) measured in the NASA Langley Grazing Flow Impedance Tube (GFIT) and Curved Duct Test Rig (CDTR). Based upon results presented in this paper the following primary conclusions are drawn:

1. When used with the closed-form and synthesized eduction data, the PGM educed impedance converges almost exactly to the known impedance of a hard wall and to that of a conventional test liner

2. When used with measured data from the Langley Grazing Flow Impedance Tube (GFIT), the PGM produces results that are consistent with that of the Wall Pressure Method (WPM)

3. Validation of the method in the Curve Duct Test Rig (CDTR) is continuing and may require the direct use of measured data without mode decomposition. Multiple options are being considered to support this approach with quasi-3D analysis. However, it appears likely that a fully 3-D code will be required to achieve robust impedance eduction in this aeroacoustic environment.

\section{Acknowledgment}

This work was funded by NASA's Subsonic Fixed Wing Project of the Fundamental Aeronautics Program.

\section{References}

\footnotetext{
${ }^{1}$ Armstrong, D. L., Beckemeyer, R. J. and Olsen, R. F., "Impedance Measurements of Acoustic Duct Liners With Grazing Flow," Paper presented at the 87th Meeting of the Acoustical Society of America, New York, NY, 1974.

${ }^{2}$ Watson, W. R., Jones, M. G., and Parrott, T. L., "Validation of an Impedance Eduction Method in Flow," AIAA Journal, Vol. 37, No. 7, pp. 818-824, July 1999 .

${ }^{3}$ Jones, M. G., Parrott, T. L., and Watson, W. R., "Comparison of Acoustic Impedance Eduction Techniques for Locally-Reacting Liners," AIAA Paper 2003-3306, May 2003.

${ }^{4}$ Elnady, T. and Boden, H., "An Inverse Analytical Method for Extracting Liner Impedance from Pressure Measurements," AIAA Paper 2004-2836, May 2004.

${ }^{5}$ Jones, M. G., Watson, W. R., Tracy, M. B., and Parrott, T. L., "Comparison of Two Waveguide Methods for Educing Liner Impedance in Grazing Flow," AIAA Journal, Vol. 42, No. 2, 2004, pp. 232-240.

${ }^{6}$ Auregan, Y., Leroux, M. and Pagneux, V., "Measurement of Liner Impedance with Flow by an Inverse Method," AIAA Paper 2004-2838, May 2004.

${ }^{7}$ Watson, W. R., Jones, M. G., and Parrott, T. L., "Comparison of a Convected Helmholtz and Euler Model for Impedance Eduction in Flow," AIAA Paper 2006-2643, May 2006.

${ }^{8}$ Elnady, T., Musharraf, M., Boden, H., and Elhadidi, B., "Validation of an Inverse Analytical Technique to Educe Liner Impedance with Grazing Flow," AIAA paper 2006-2639, May 2006.

${ }^{9}$ Watson, W., Jones, M., and Parrott, T., "Investigation of an Anomaly Observed in Impedance Eduction Techniques," AIAA Paper 2008-3013, May 2008.

${ }^{10}$ Jing, X., Peng, S., and Sun, X., “A Straightforward Method for Wall Impedance Eduction in a Flow Duct,” JASA, Vol. 124, No. 1, July 2008.

${ }^{11}$ Simonich, J., Narayanan, S., Morin, B., and Patrick, W., "Development and Quantification of an In-Situ Grazing Flow Impedance Measurement Technique," AIAA Paper 2005-2848, May 2005.

${ }^{12}$ Jones, M. G., Watson, W. R., Parrott, T. L., and Smith, C. D., "Design and Evaluation of Modifications to the NASA Langley Flow Impedance Tube," AIAA Paper 2004-2837, May 2004.
} 
${ }^{13}$ Gerhold, C., Cabell, R., and Brown, M., "Development of an Experimental Rig for Investigation of Higher Order Modes in Ducts," AIAA Paper 2006-2637, May 2006.

${ }^{14}$ Jones, M. G., Watson, W. R., and Nark, D. M., "Optimization of Acoustic Pressure Measurements for Impedance Eduction ," AIAA Paper 2007-3531, May 2007.

${ }^{15}$ Watson, W. R. and Jones, M. G, “Validation of a New Procedure for Impedance Eduction in Flow,” AIAA Paper 2010-3764, June 2010.

${ }^{16}$ Myers, M. K., "On the Acoustic Boundary Condition in the Presence of Flow," JSV, Vol. 71, No. 3, 1980, pp.429-434.

${ }^{17}$ Davidon, W. C., "Variable Metric Methods for Minimization," Argonne National Labs Report ANL-5990, 1959.

${ }^{18}$ Fletcher, R. and Powell, M. J. D., “A Rapidly Convergent Descent Method for Minimization,” The Computer Journal, Vol. 6, No. 2, 1963, pp. $163-168$.

${ }^{19}$ Stewart, G. W., III, “A Modification of Davidon's Minimization Method to Accept Difference Approximations of Derivatives," Journal of ACM, Vol. 14, No. 1, 1967, pp 72-83.

${ }^{20}$ Snider, Royce: Attenuation Predictions for Segmented Liners in Supersonic Flow, M.S, Thesis, The George Washington University, July 1993.

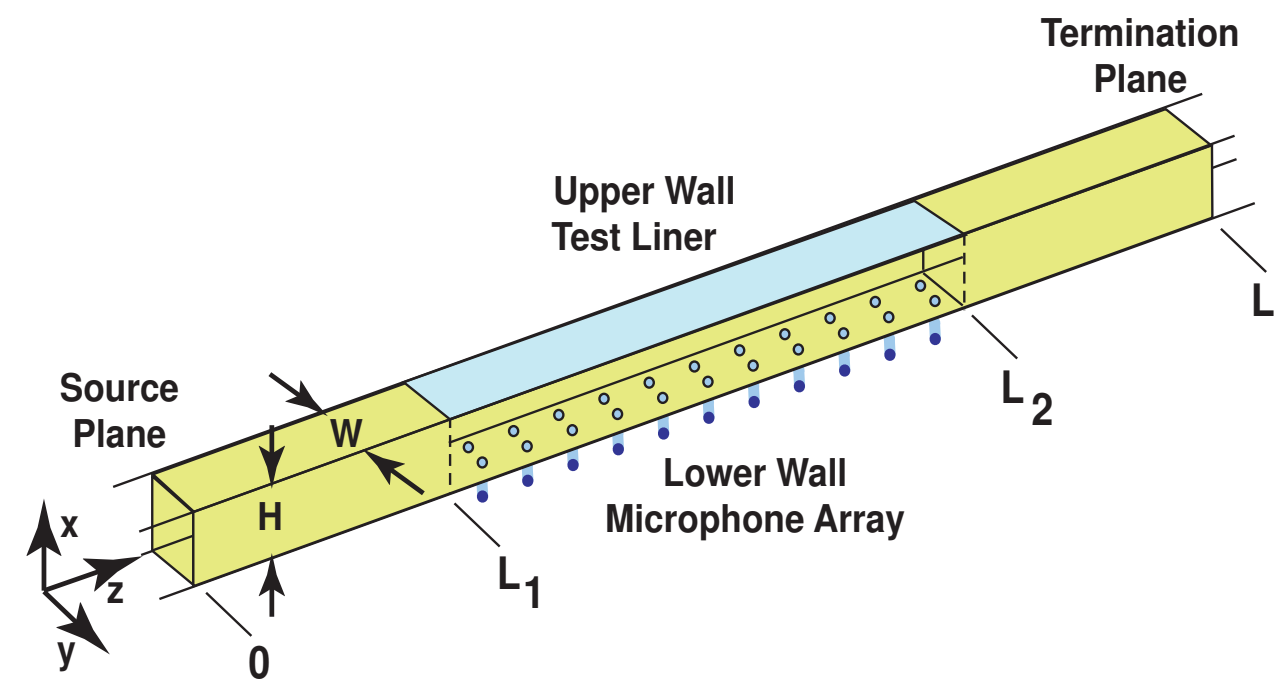

Figure 1. Schematic of the GFIT with microphones embedded in the liner test section.

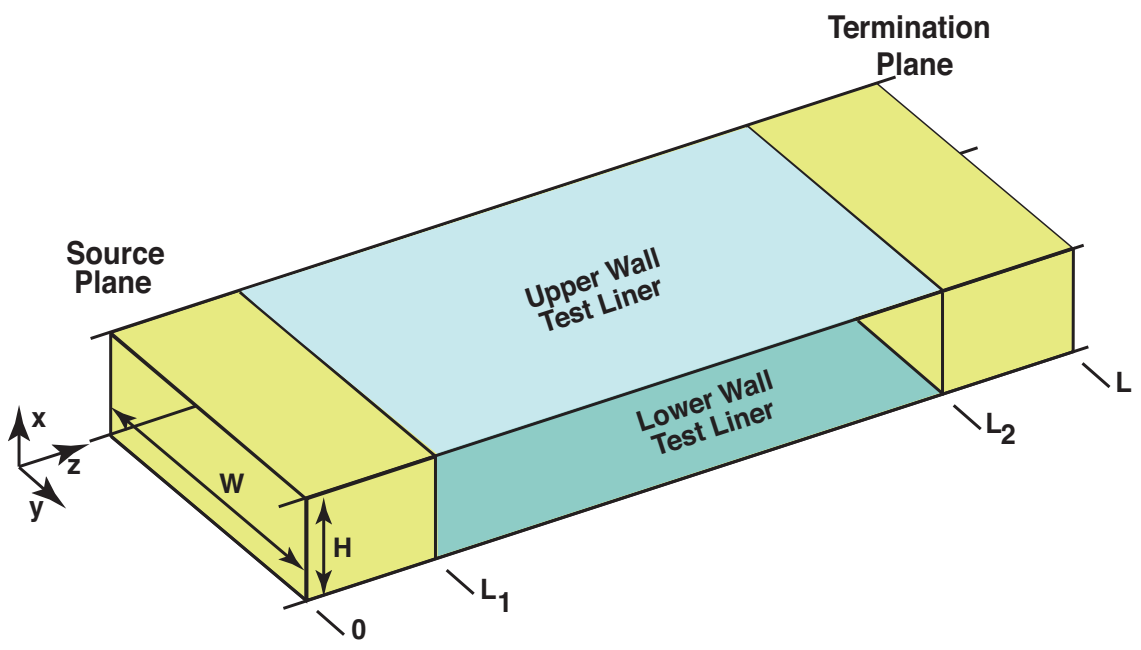

Figure 2. Schematic of the CDTR flow duct with microphones removed from liner test section. 


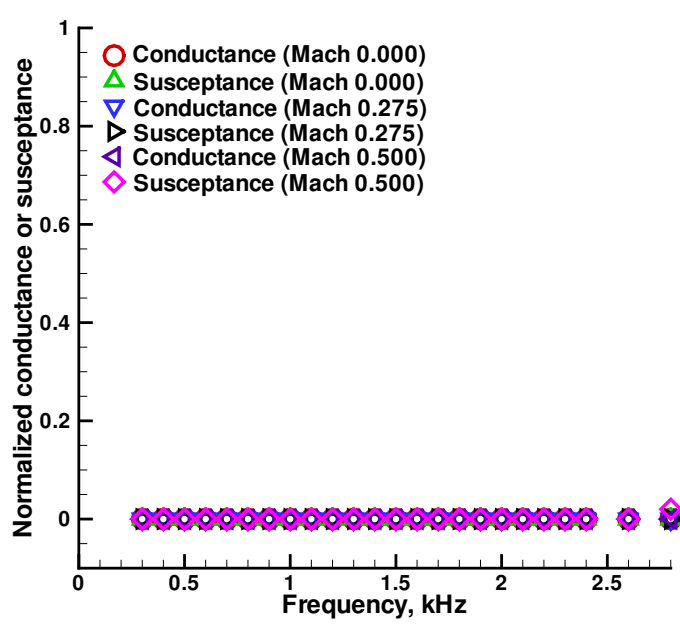

(a) Plane wave mode (00)

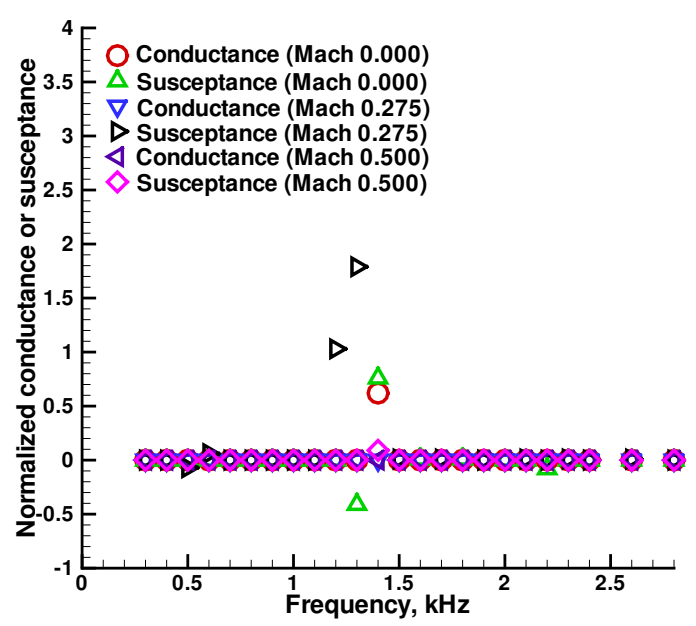

(b) Higher order mode (01)

Figure 3. PGM educed conductance and susceptance for plane wave and higher-order mode in a hard wall duct .

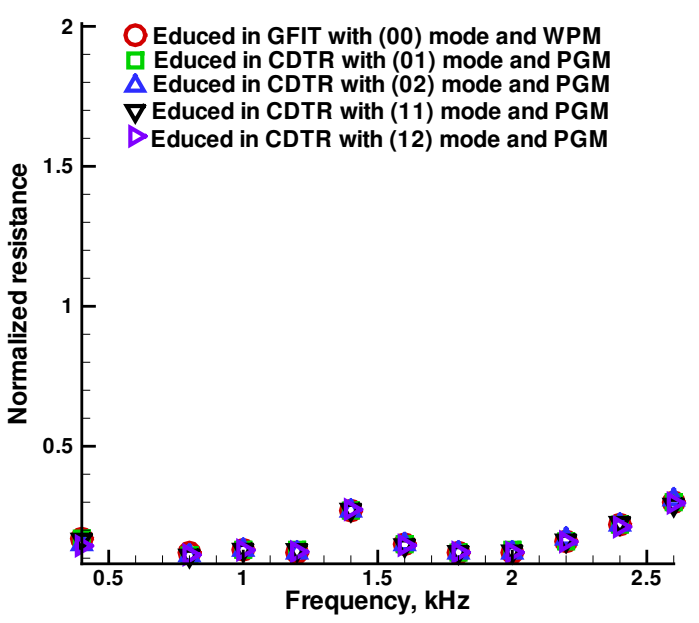

(a) Normalized resistance

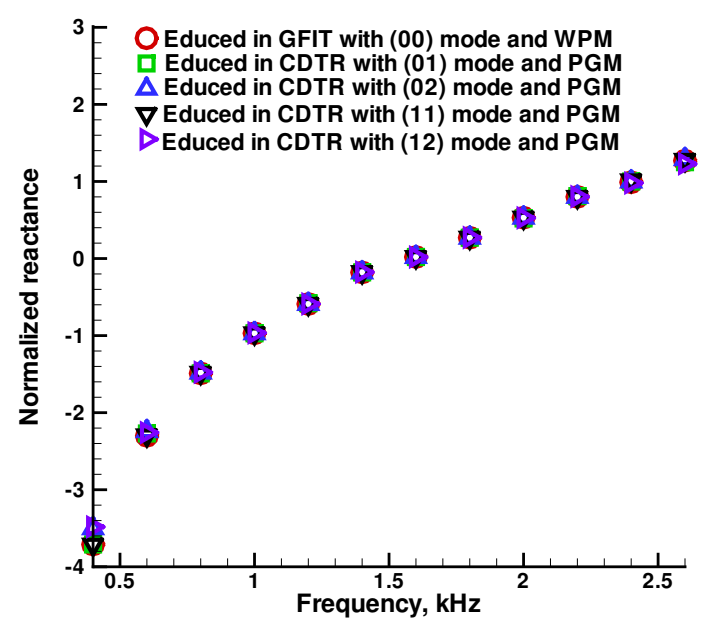

(b) Normalized reactance

Figure 4. PGM educed normalized resistance and reactance for conventional liner for several higher order mode sources with flow off. 


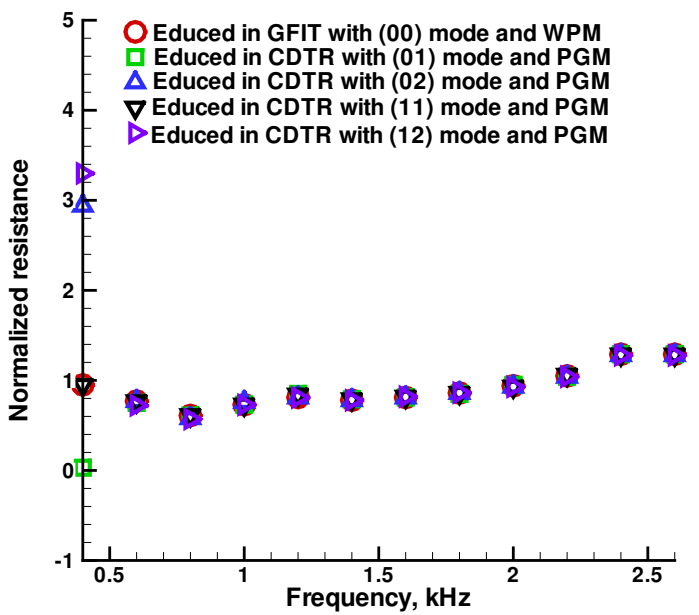

(a) Normalized resistance at Mach 0.275

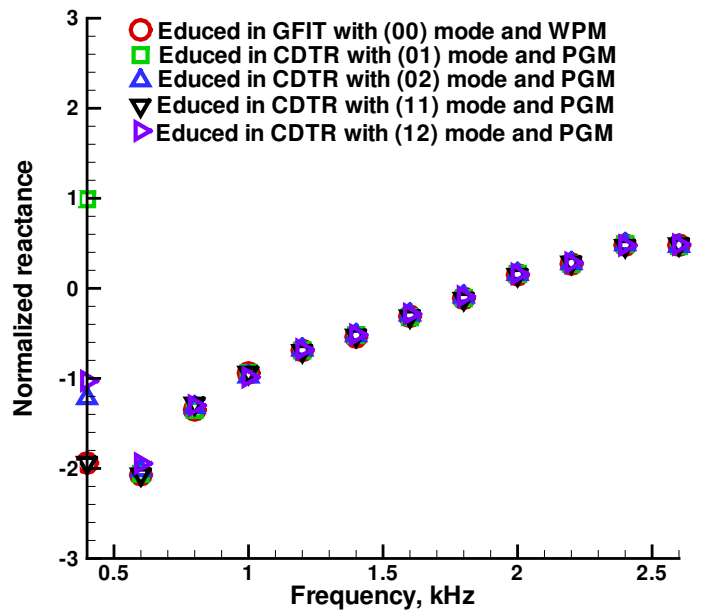

(b) Normalized reactance at Mach 0.275

Figure 5. PGM educed normalized resistance and reactance for conventional liner for several higher order mode sources at Mach 0.275 .

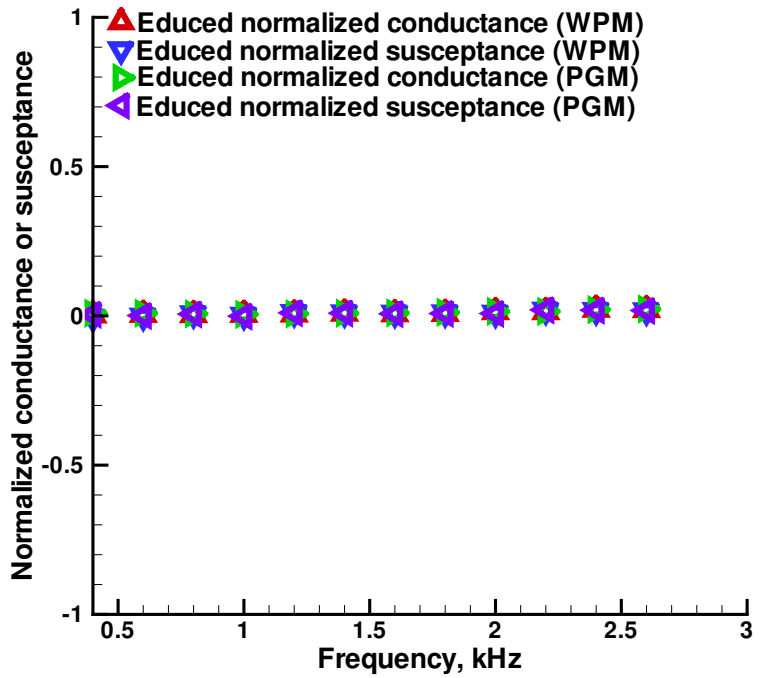

Figure 6. Educed normalized conductance and susceptance of the hard wall insert for the flow-off condition. 


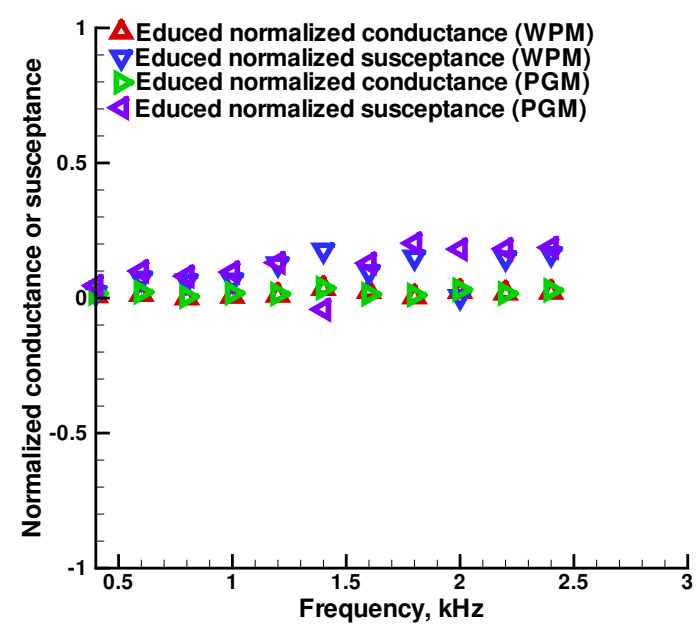

(a) Mach 0.300

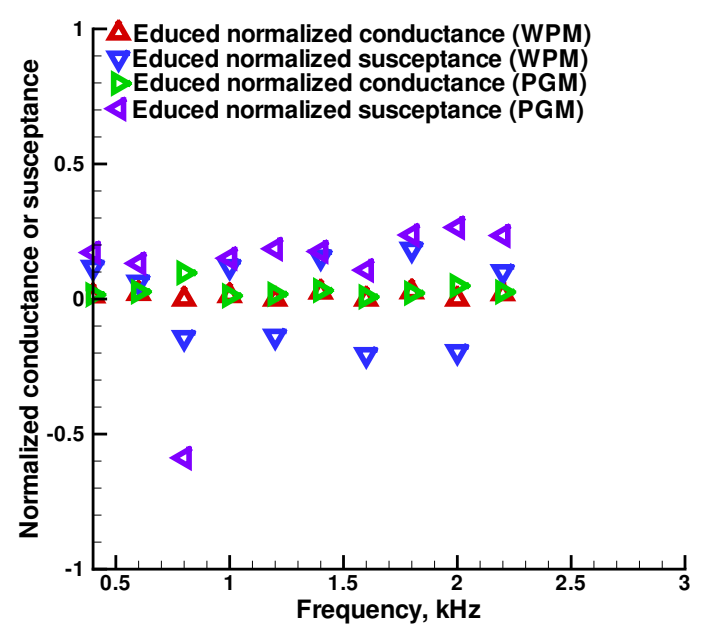

(b) Mach 0.500

Figure 7. Educed normalized conductance and susceptance of the hard wall insert for the flow-on conditions.

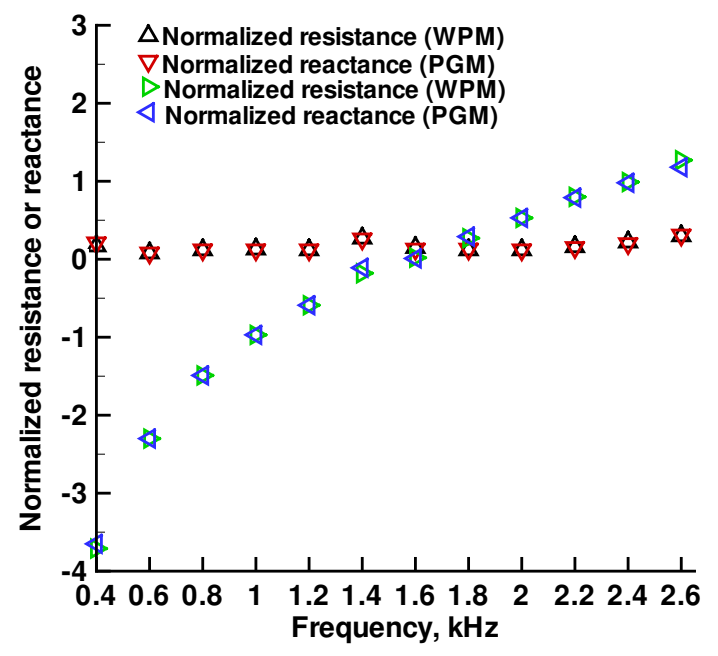

Figure 8. Educed normalized resistance and reactance of the conventional liner for the flow-off condition. 


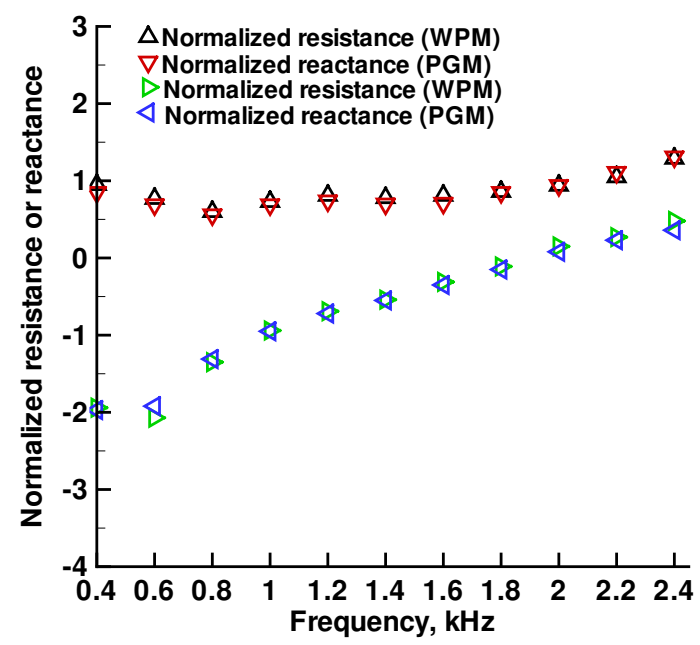

(a) Mach 0.300

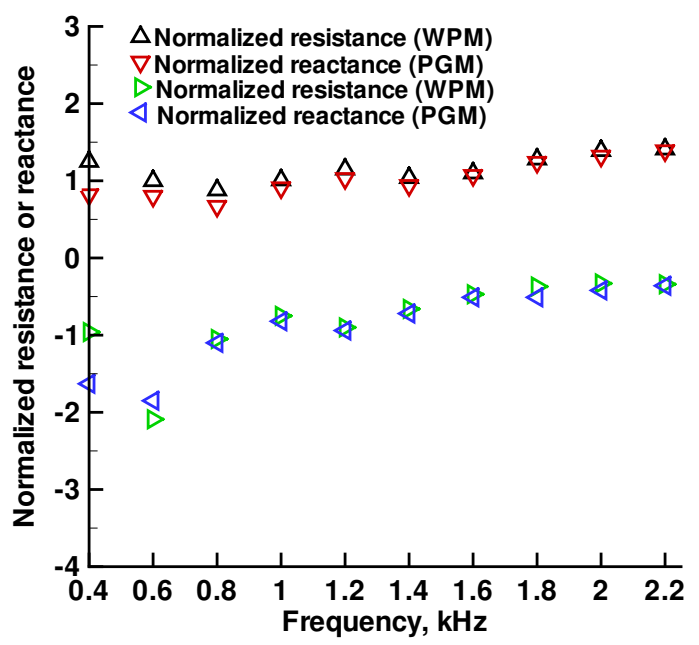

(b) Mach 0.500

Figure 9. Educed normalized resistance and reactance of the conventional liner for the flow-on conditions.

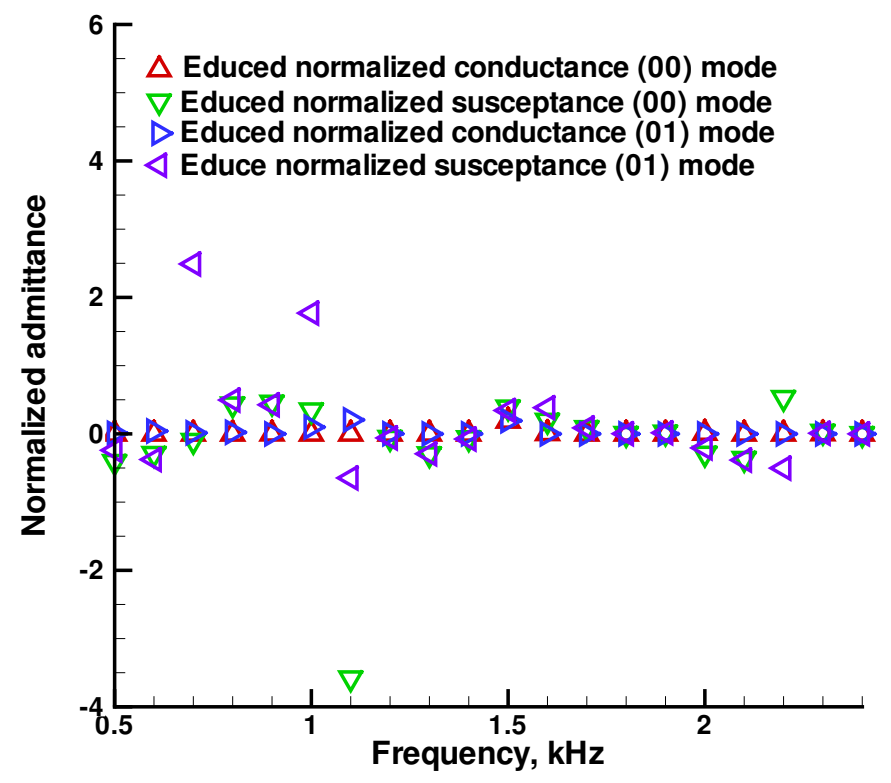

Figure 10. Educed normalized conductance and susceptance using the PGM for a hard wall insert in the CDTR with flow off . 